\title{
Adapting a Virtual Agent's Conversational Behavior by Social Strategies
}

\author{
Nikita Mattar and Ipke Wachsmuth \\ Artificial Intelligence Group, Bielefeld University \\ Universitätsstr. 25, 33615 Bielefeld, Germany \\ [nmattar|ipke]@techfak.uni-bielefeld.de
}

\begin{abstract}
Interpersonal encounters are a complex phenomenon in human-human interaction. As social encounters with virtual agents become more important, these agents have to cope with problems of social perception, as well. To account for tasks concerned with the acquisition, utilization, and recall of social information, we earlier proposed to equip virtual agents with a Person Memory. In this paper we present how information available through a Person Memory enables the conversational agent Max to tackle different interpersonal situations.
\end{abstract}

Keywords: conversational agents, intelligent virtual agents, human-agent interaction, person memory, interpersonal encounters, social information, person perception

\section{Introduction}

In intelligent virtual agent research an important goal is to create agents that are as believable as possible, motivated by the advancement of virtual agents from tools to human-like partners. For instance, so called companion agents are envisioned to interact over a long period of time with, or even beyond the lifetime of, their owners [8], [7]. Human-like memory systems, i.e., autobiographic and episodic memories, are employed to improve believability of such agents [3], [2]. However, these systems focus on the agent's own experiences. As conversational agents start to appear in everyday interaction scenarios, the question arises if such egocentric memory systems are sufficient to handle requirements that come up in social encounters. To be accepted as human-like conversational partners, virtual agents may have to cope with various interpersonal situations. For instance, in initial encounters altruistic behavior may seem appropriate for a companion agent, whereas a slightly selfish behavior may be more adequate in situations where a companion agent acts on the behalf of its owner.

In this paper we present how information of a model of Person Memory can be exploited to adapt a virtual agent to its interlocutors according to different social situations. In the following, social memory tasks and social memory strategies used in the model are described. Brief sample dialogs demonstrate how the Person Memory can be exploited to tackle social encounters by using social strategies: an altruistic, a balanced, and a selfish strategy. The paper concludes with an outlook on future work. 


\section{Adapting Conversational Behavior by Social Strategies}

In order to examine and grasp effects of so called human person perception - the acquisition, utilization, and recall of social information during human interpersonal encounters -, we proposed to equip virtual agents with a Person Memory [5]. To exploit information provided by the Person Memory in social situations, Social Memory Tasks and Social Strategies are a crucial part of our model. Social Memory Tasks define how the information is exploited. They are divided into core and extended tasks. Core tasks handle basic actions, like storage of new, or retrieval of existing, information. Extended tasks carry out more context based information retrieval and manipulation on the data, like calculating probabilities for the use of dialog sequences ("Question/Answer" vs. more complex sequences like "Question/Counter/Probe/Reply", cf. [6]), or selecting a topic according to different categories ( "communication", "immediate", "external", cf. [1]). Furthermore, extended tasks can be exchanged dynamically at run time. This allows to define tasks including different information, for instance, when selecting a topic the agent should bring up during conversation.

Social strategies are used in this model of Person Memory to activate tasks appropriate for a situation. So far, they consist of a trigger that is sensitive to a certain social situation and a mapping of social memory tasks to keywords that are used to identify tasks within the Person Memory. When a strategy is activated by a situation, its associated tasks are registered within the Person Memory. The registered tasks are used subsequently when a task with an according keyword is scheduled for execution. Algorithm 1 depicts a sample implementation of a social memory task as used by a balanced social strategy. Here, a topic for conversation is selected according to the information of the interlocutor and the agent that is available in the Person Memory. An altruistic, or a selfish, task can be obtained from the balanced task by returning the interest of the interlocutor, or the agent, respectively.

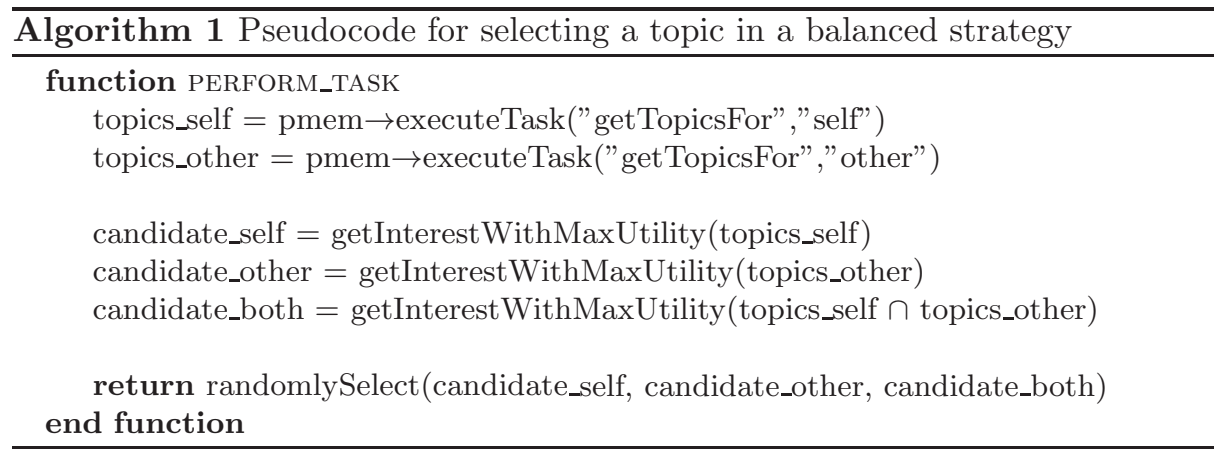

This way, information contained in the Person Memory, together with strategies and accompanying tasks that influence how the information is exploited, allow for an adaptation of the agent to different social encounters. 


\section{Sample Dialogs}

The following dialog excerpts (translated to English) demonstrate how one aspect - choice of utterances - of the conversational style of the agent Max [4] is adapted by the use of social strategies. While the current implementation allows to adapt further aspects, the capabilities of the Person Memory can be demonstrated here by adapting a single feature (yet the resulting dialogs may appear somewhat superficial).

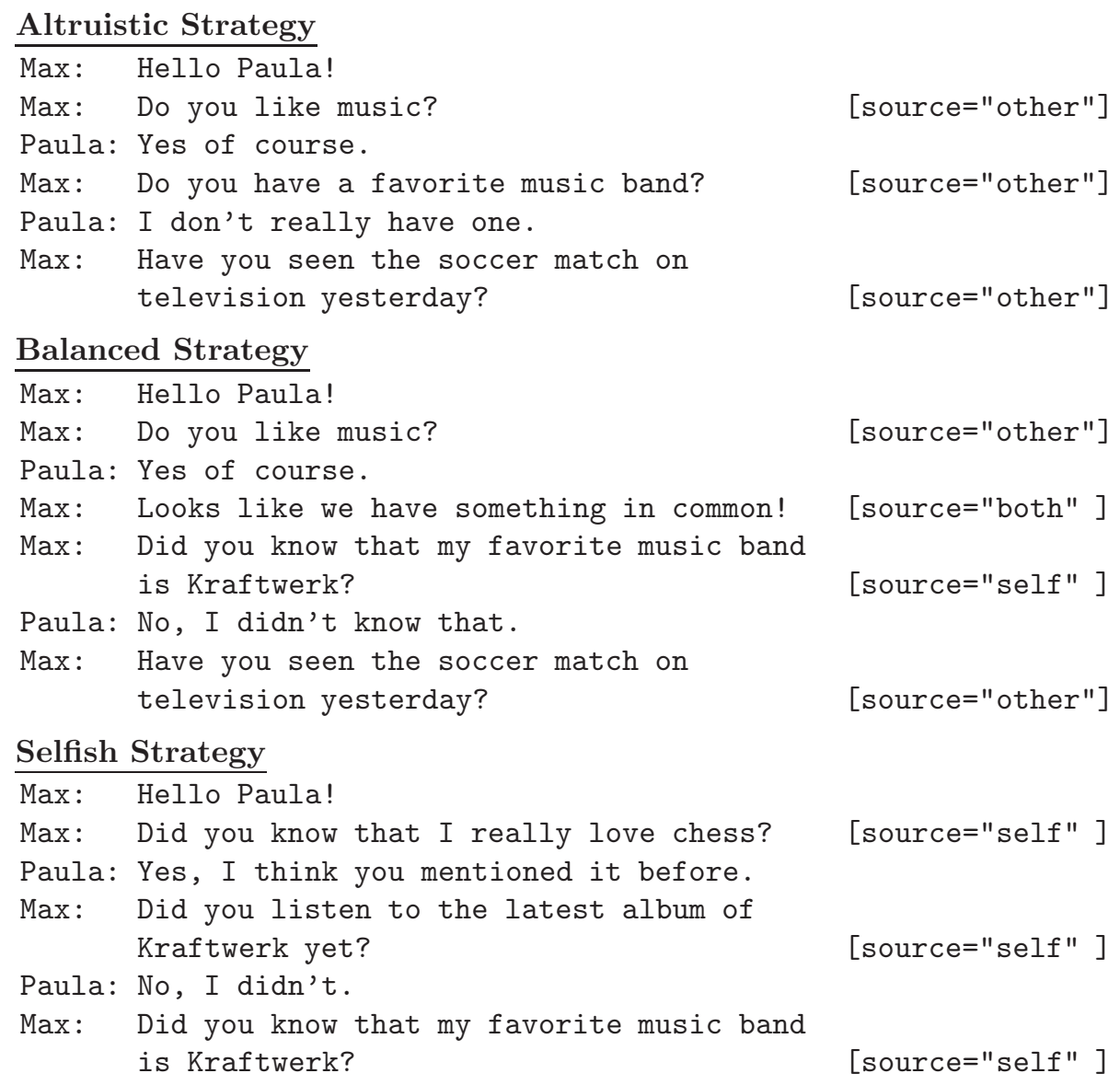

\subsection{Discussion}

The possibility to change between memory tasks based on different social strategies allows Max to adapt to different application scenarios. In the first case an altruistic strategy is used, so Max focuses on the interests of its interlocutor and avoids remarks about own interests. When using the balanced strategy, Max selects interests of the interlocutor as well as of his own as topics for conversation. In the third example, Max completely ignores his interlocutor's interests. 
In contrast to the first case, interests of the agent are only selected as topics for conversation when using a selfish strategy.

\section{Future Work}

In this paper, we demonstrated how information contained in a model of Person Memory can be utilized to adapt a virtual agent's behavior according to social strategies. An evaluation of the model will be conducted when the Person Memory is ready to be deployed in a real-life application, like the museum setting the agent Max already operates in. More aspects of conversational behavior than touched upon in this paper will be adapted, e.g., the dialog structure and topic categories (cf. 2). By that, we expect to obtain hints on promising combinations that lead to more natural dialogs in different social settings.

\section{Acknowledgments}

This paper is a preprint version of an article published by Springer-Verlag. The original publication is available at http://link.springer.com/chapter/10. $1007 \% 2 F 978-3-642-40942-4 \_26$.

\section{References}

1. Breuing, A., Wachsmuth, I.: Let's Talk Topically with Artificial Agents! Providing Agents with Humanlike Topic Awareness in Everyday Dialog Situations. In: ICAART 2012 - Proceedings of the 4th International Conference on Agents and Artificial Intelligence. pp. 62-71. SciTePress (2012)

2. Brom, C., Lukavský, J., Kadlec, R.: Episodic Memory for Human-like Agents and Human-like Agents for Episodic Memory. International Journal of Machine Consciousness 2(02), 227-244 (2010)

3. Ho, W., Dautenhahn, K.: Towards a Narrative Mind: The Creation of Coherent Life Stories for Believable Virtual Agents. In: Prendinger, H., Lester, J., Ishizuka, M. (eds.) Intelligent Virtual Agents, Lecture Notes in Computer Science, vol. 5208, pp. 59-72. Springer Berlin Heidelberg (2008)

4. Kopp, S., Gesellensetter, L., Krämer, N.C., Wachsmuth, I.: A Conversational Agent as Museum Guide - Design and Evaluation of a Real-World Application. In: Panayiotopoulos, T., Gratch, J., Aylett, R., Ballin, D., Olivier, P., Rist, T. (eds.) Intelligent Virtual Agents, Lecture Notes in Computer Science, vol. 3661, pp. 329-343. Springer Berlin Heidelberg (2005)

5. Mattar, N., Wachsmuth, I.: A Person Memory for an Artificial Interaction Partner. In: Proceedings of the KogWis 2010. pp. 69 - 70 (2010)

6. Mattar, N., Wachsmuth, I.: Small Talk Is More than Chit-Chat - Exploiting Structures of Casual Conversations for a Virtual Agent. In: Glimm, B., Krüger, A. (eds.) KI 2012: Advances in Artificial Intelligence, Lecture Notes in Computer Science, vol. 7526, pp. 119-130. Springer Berlin Heidelberg (2012) 
7. O'Hara, K.: Arius in cyberspace: Digital Companions and the limits of the person. In: Wilks, Y. (ed.) Close Engagements with Artificial Companions: key social, psychological, ethical and design issues, p. 68ff. John Benjamins Publishing Company (2010)

8. Wilks, Y.: Artificial Companions as a new kind of interface to the future Internet. Research Report 13, Oxford Internet Institute/University of Sheffield (2006) 